\title{
The genome of the soybean cyst nematode (Heterodera glycines) reveals complex patterns of duplications involved in the evolution of parasitism genes
}

\author{
Rick Masonbrink ${ }^{1,2}$, Tom R. Maier ${ }^{1}$, Usha Muppirala ${ }^{1,2}$, Arun S. Seetharam ${ }^{1,2}$, Etienne Lord ${ }^{3}$, Parijat S. Juvale ${ }^{1}$, \\ Jeremy Schmutz ${ }^{4,5}$, Nathan T. Johnson ${ }^{6}$, Dmitry Korkin ${ }^{6,7}$, Melissa G. Mitchum ${ }^{8}$, Benjamin Mimee ${ }^{3}$, \\ Sebastian Eves-van den Akker ${ }^{9}$, Matthew Hudson ${ }^{10}$, Andrew J. Severin ${ }^{2 *}$ and Thomas J. Baum ${ }^{1}$
}

\begin{abstract}
Background: Heterodera glycines, commonly referred to as the soybean cyst nematode (SCN), is an obligatory and sedentary plant parasite that causes over a billion-dollar yield loss to soybean production annually. Although there are genetic determinants that render soybean plants resistant to certain nematode genotypes, resistant soybean cultivars are increasingly ineffective because their multi-year usage has selected for virulent $\mathrm{H}$. glycines populations. The parasitic success of $\mathrm{H}$. glycines relies on the comprehensive re-engineering of an infection site into a syncytium, as well as the long-term suppression of host defense to ensure syncytial viability. At the forefront of these complex molecular interactions are effectors, the proteins secreted by $\mathrm{H}$. glycines into host root tissues. The mechanisms of effector acquisition, diversification, and selection need to be understood before effective control strategies can be developed, but the lack of an annotated genome has been a major roadblock.

Results: Here, we use PacBio long-read technology to assemble a H. glycines genome of 738 contigs into $123 \mathrm{Mb}$ with annotations for 29,769 genes. The genome contains significant numbers of repeats (34\%), tandem duplicates (18.7 Mb), and horizontal gene transfer events (151 genes). A large number of putative effectors (431 genes) were identified in the genome, many of which were found in transposons.

Conclusions: This advance provides a glimpse into the host and parasite interplay by revealing a diversity of mechanisms that give rise to virulence genes in the soybean cyst nematode, including: tandem duplications containing over a fifth of the total gene count, virulence genes hitchhiking in transposons, and 107 horizontal gene transfers not reported in other plant parasitic nematodes thus far. Through extensive characterization of the $\mathrm{H}$. glycines genome, we provide new insights into $\mathrm{H}$. glycines biology and shed light onto the mystery underlying complex host-parasite interactions. This genome sequence is an important prerequisite to enable work towards generating new resistance or control measures against $H$. glycines.
\end{abstract}

Keywords: Heterodera glycines, SCN, Soybean cyst nematode, Genome, Tandem duplication, Effector, Evolution

\footnotetext{
* Correspondence: severin@iastate.edu

${ }^{2}$ Genome Informatics Facility, lowa State University, Ames, IA, USA

Full list of author information is available at the end of the article
}

(c) The Author(s). 2019 Open Access This article is distributed under the terms of the Creative Commons Attribution 4.0 International License (http://creativecommons.org/licenses/by/4.0/), which permits unrestricted use, distribution, and reproduction in any medium, provided you give appropriate credit to the original author(s) and the source, provide a link to the Creative Commons license, and indicate if changes were made. The Creative Commons Public Domain Dedication waiver (http://creativecommons.org/publicdomain/zero/1.0/) applies to the data made available in this article, unless otherwise stated. 


\section{Background}

The soybean cyst nematode (SCN) Heterodera glycines is considered the most damaging pest of soybean and poses a serious threat to a sustainable soybean industry [1]. H. glycines management relies on crop rotations, nematode resistant crop varieties, and a panel of biological and chemical seed treatments. However, cyst nematodes withstand adverse conditions and remain dormant for extended periods of time, and therefore, are difficult to control. Furthermore, the overuse of resistant soybean varieties has stimulated the proliferation of virulent nematode populations that can infect these varieties [2]. Hence, there continues to be a strong need to identify, develop, and implement novel sources of nematode resistance and management strategies.

$H$. glycines nematodes are obligate endoparasites of soybean roots. Once they emerge from eggs in the soil, they find nearby soybean roots and penetrate the plant tissue where they migrate in search for a suitable feeding location near the vascular cylinder. The now sedentary $H$. glycines convert adjacent root cells into specialized, fused cells that form the feeding site, termed syncytium [3]. The parasitic success of $H$. glycines depends on the formation and long-term maintenance of the syncytium, which serves as the sole source of nutrition for the remainder of its life cycle. Host finding, root penetration, syncytium induction, and the long-term successful suppression of host defenses are all examples of adaptation to a parasitic lifestyle. At the base of these adaptations lies a group of nematode proteins that are secreted into plant cells to modify host processes [4]. Intense research is focused on identifying these proteins, called effectors, and to elucidate their complex functions. To date, over $80 \mathrm{H}$. glycines effectors have been identified and confirmed $[5,6]$, although many more remain to be discovered. Characterization of some known effectors has provided critical insights into the parasitic strategies of $H$. glycines. For example, these studies revealed that effectors are involved in a suite of functions, including defense suppression, plant hormone signaling alteration, cytoskeletal modification, and metabolic manipulation (reviewed by [7-10]). However, research has yet to provide a basic understanding of the molecular basis of virulence, i.e., the ability of some nematode populations to infect soybean plants with resistance genes, while other nematode populations are controlled by these resistance genes.

H. glycines populations are categorized into $\mathrm{Hg}$ types based on their virulence to a panel of soybean cultivars with differing resistance genetics $[2,11]$. Based on the $\mathrm{Hg}$ type designation, growers can make informed decisions on soybean cultivar choice. To date, the $\mathrm{Hg}$ type designation can only be ascertained through time-consuming and expensive greenhouse experiments. However, once the genetic basis for virulence phenotypes has been explored, it is conceivable that molecular tests can be developed to make $\mathrm{Hg}$ type identification fast and reliable.

Resistant soybean cultivars are becoming less effective, as $H$. glycines populations alter their $\mathrm{Hg}$ type designation as a function of the soybean resistance genes to which the nematode population is exposed. In other words, when challenged with a resistant soybean cultivar for an extended duration, the surviving nematodes of an otherwise largely non-virulent $H$. glycines population will eventually shift towards a new $\mathrm{Hg}$ type that is virulent on resistant soybean cultivars [2]. It is unknown if this phenomenon solely relies on the selection of virulent genotypes already present within a given nematode population, or if $H$. glycines wields the power to diversify an existing effector portfolio to quickly infect resistant soybean cultivars. In addition, such genetic shifts appear to be distinct across populations with the same pathotype, indicating populations can independently acquire the ability to overcome host resistance [12]. Understanding these and other questions targeting the molecular basis of $H$. glycines virulence are critical for sustainable soybean production in a time when virulent nematodes are becoming more prevalent.

Scientists can finally start answering such questions, as we are presenting a near-complete genome assembly and extensive effector annotation of $H$. glycines, along with single-nucleotide polymorphisms (SNPs) associated with fifteen $H$. glycines populations of differing virulence phenotypes. PacBio long-reads were assembled and annotated into 738 contigs of $123 \mathrm{Mb}$ containing 29,769 genes. The $H$. glycines genome has significant numbers of repeats (34\% of the genome), tandem duplications (14.6 Mb), and horizontal gene transfer events (151 genes). Using this genome, we explored potential mechanisms for how effectors originate, duplicate, and diversify. Specifically, we found that effectors are frequently associated with tandem duplications, DNA transposons, and LTR retrotransposons. Additionally, we have leveraged RNA-seq data from pre-parasitic and parasitic nematodes and DNA sequencing across $15 \mathrm{H}$. glycines populations to further characterize effector expression and diversity.

\section{Results}

Genome assembly, annotation, and completeness

$H$. glycines genomic DNA was extracted, and PacBio sequencing generated 2.4 million subreads with an average length of $7.6 \mathrm{~kb}$ corresponding to a coverage of $141 \times$ at an estimated genome size of $129 \mathrm{MB}$ [13]. Due to the high level of heterozygosity of $H$. glycines populations, our early PacBio-only assemblies using Falcon and Falcon-Unzip resulted in an abundance of heterozygous contigs (haplotigs). Therefore, we reduced the 
heterozygosity of the original reads using a combination of Falcon, CAP3, and manual scaffolding of the assembly graph in Bandage. The final assembly was polished with Quiver and contains 738 contigs with an N50 of $304 \mathrm{~kb}$ and a total genomic content of 123,846,405 nucleotides (Fig. 1). We confirmed the assembly to be free of contamination using Blobtools (4.8.2) (Additional file 1: Figure S1) and validated for completeness by alignment of raw data: $88 \%$ of the RNA-seq [14] and 93\% of the PacBio preads (Additional file 1: Table S1). In addition, approximately $72 \%$ of the 982 Nematoda-specific BUSCO genes are complete in the $H$. glycines genome, which is comparable to BUSCO scores in other Tylenchida genomes (Additional file 1: Table S2). Remarkably, only $56 \%$ of the BUSCO genes in $H$. glycines are single-copy, while $16 \%$ were duplicated, a statistic that is comparable to the allopolyploid root-knot nematode Meloidogyne incognita (Additional file 1: Table S2) [15-17]. Synteny diminished as phylogenetic relatedness declined (Fig. 1, Additional file 1: Figures S2-S6), supporting the established phylogeny alongside a phylogenetic tree (Fig. 1) derived from 651/982 single-copy BUSCO genes shared by at least three species among $H$. glycines, Globodera pallida, Globodera ellingtonae, Globodera rostochiensis,
Meloidogyne hapla, M. incognita, and Bursaphelenchus xylophilus.

Gene annotations were performed using Braker on an unmasked assembly, as multiple known effector alignments were absent from predicted genes when the genome was masked (Additional file 1: Figure S7). While all known effectors are present in the assembly, the resulting gene count of 29,769 also includes many expressed repetitive elements $(12,357)$. A variety of transcriptional sequencing was used as input for gene annotations, including 230 million RNA-seq reads from both pre-parasitic and parasitic J2 H. glycines nematodes [14], 34,041 iso-seq reads from early, middle and late life stages of both a virulent and an avirulent strain, and the entirety of the $H$. glycines ESTs in NCBI $(35,796)$.

\section{Effector gene identification}

Effector genes give rise to proteins that are secreted into the host to modify host cellular processes. Many effectors originate in the esophageal glands. Dorsal gland-expressed genes (DOGs) are mostly active during the later parasitic stages when syncytial development is initiated and progressing. In Globodera cyst nematode species, a putative regulatory promoter motif of dorsal

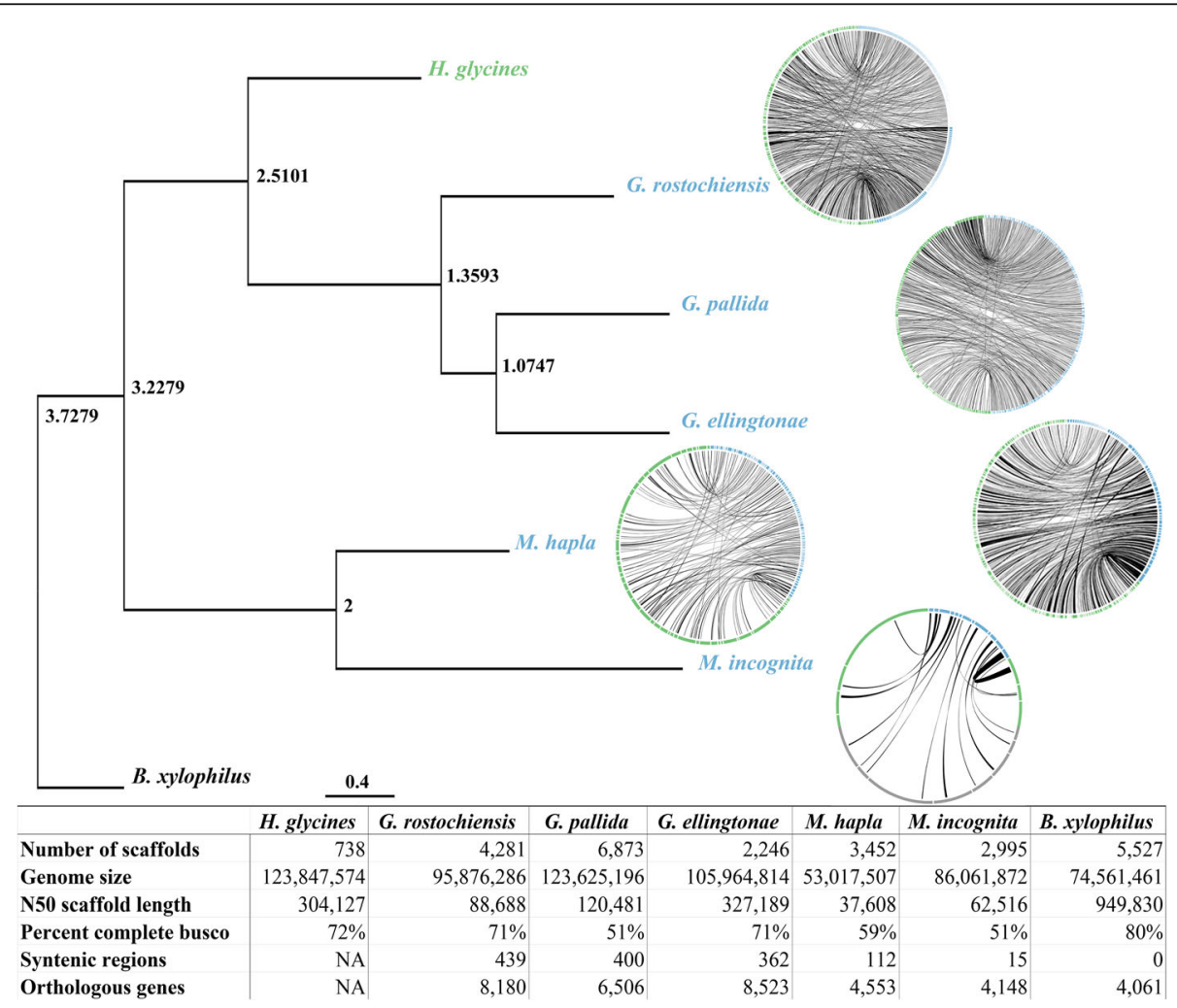

Fig. 1 Phylogenetic relationships of species related to H. glycines. Phylogenetic tree of BUSCO genes with synteny representing the relatedness of each species. Synteny is inversely correlated with phylogenetic distance, as syntenic multiplicons decrease from hundreds in Globodera species, to zero in $B$. xylophilus, Green contigs denote H. glycines, while blue represent the respective related species. Node labels represent node ages. Pertinent comparative genome stats are found in the footer 
gland cell expression, the DOG box, was recently identified [12]. To determine whether the regulation of dorsal gland cell expression in Heterodera species may be under similar control, we generated a non-redundant list of putative homologues of known dorsal gland effectors from cyst nematodes. This included all known dorsal gland effectors, a large family of recently characterized glutathione synthetase-like effectors [18], and all DOG-box associated effectors of G. rostochiensis. A total of 128 unique dorsal gland effector-like loci were identified in the genome, their promoter regions were extracted and compared to a random set of non-effector gene promoters using a non-biased differential motif discovery algorithm. Using this approach, a near-identical DOG box motif was identified (Fig. 2a), enriched on both strands of dorsal gland effector-like loci promoters approximately $100-150 \mathrm{bp}$ upstream of the start codon (Fig. 2b). DOG box motifs occur at a greater frequency in promoters than expected by random, however their presence in a promoter is only a modest prediction of secretion (Fig. 2c). Taken together this suggests that the cis-regulatory elements controlling dorsal gland effector expression may be a conserved feature in cyst nematodes, predating at least the divergence of Globodera and Heterodera over 30 million years ago.

Given that DOG boxes are only present in some effector promoters, to identify a comprehensive repertoire of effectors we combined several methods and criteria. First, we aligned the 80 known $H$. glycines effector sequences to the genome using GMAP, identifying 121 putative effector genes. Second, the same 80 known effectors were subjected to motif discovery with MEME, identifying 24 motifs in 60/80 effectors (Additional file 1: Figure S8). One motif (motif 1) was a known signal peptide found in 10/60 effectors [19]. In addition, motifs 8,12 , and 18 were also consistently found at the $\mathrm{N}$ terminus in $7 / 60,16 / 60$, and $17 / 60$ effectors, respectively. Because genes containing these motifs may also be effectors, the 24 motifs (Additional file 2: Data S1) were queried against the $H$. glycines predicted proteome using FIMO, revealing a set of 292 proteins with at least one effector-like motif. All three effector gene predictions were merged to produce a unique set of 431 effector-like genes. This gene set was used in downstream analyses exploring effector evolution. Of the 431 effector-like loci, 216 are predicted to encode a secretion signaling peptide and lack a transmembrane domain. While the remaining 215 effector-like loci may contain non-effectors, they were retained for downstream evolutionary analyses because they may represent genes with non-canonical secretion signals, "progenitor" housekeeping genes that gave rise to effectors (e.g. GS-like effectors [18], SPRY-SECs [20], etc.), or an effector graveyard.
Horizontal gene transfer (HGT) was important for the evolution of parasitism in the root-knot and cyst nematodes [21-28]. To better understand the role of HGT in the evolution of effectors in $H$. glycines, we calculated an Alien Index (AI) for each transcript using a ratio of similarity to metazoan and non-metazoan sequences [29]. A total of 1678 putative HGT events (AI>0) were observed in the predicted $H$. glycines proteome (Additional file 3: Data S2), distributed on 461 different contigs (Fig. 3a). This prediction includes 151 genes with strong HGT support (AI> 30) (Fig. 3b), 82 genes previously identified in closely related nematodes (Additional file 1: Table S3), and 107 putative HGT reported here for the first time in plant parasitic nematodes (Additional file 4: Data S3). The number of introns was significantly reduced in genes with $\mathrm{AI}>0$ (6.8 vs 9.7, $p<0.001$, Student's t-test) (Fig. 3b), further supporting a non-metazoan origin. Among these, the highest E-values were of bacterial, fungal, or plant origin for $70.8 \%(114 / 161), 19.3$, and 9.9\%, respectively (Additional file 3: Data S2). Interestingly, only 7/151 high confidence HGT genes were co-identified as one of the 431 effector-like loci.

\section{Genomic insights into the mechanisms of effector duplication and diversification}

The tandem duplication (TD) of genes in pathogen genomes is a common evolutionary response to the arms race between pathogen and host as a means to avoid/ overcome host resistance [30]. To identify the role tandem duplications play in the duplication virulence genes, we implemented RedTandem to survey the $H$. glycines genome. We determined that a total of $18.7 \mathrm{MB}$ of the genome is duplicated with a total of 20,577 duplications in the genome. While most individual duplications were small, the average tandem duplication size was $909 \mathrm{bp}$. We verified that tandem duplications were not assembly artifacts by aligning the PacBio preads to the genome and confirmed that the larger than average tandem duplications (4410/4241) were spanned by PacBio preads across $>90 \%$ of tandem duplication length. The density of genes in the tandemly duplicated regions is higher than in non-duplicated regions of the genome: 6730/ 18.7 MB ( 360 genes/MB) vs 23,039/105.2 MB ( 219 genes/MB), and thus contributes to one fifth of the total gene count in the $H$. glycines genome. The largest groups of orthologous genes found in tandem duplications $(881 / 3940$ genes) were annotated with BLAST to the NCBI non-redundant (NR) database, revealing that the 38 largest clusters of duplicated genes were frequently transposable element genes, effector/gland-expressed genes, or $\mathrm{BTB} / \mathrm{POZ}$ domain-containing genes (Additional file 1: Figure S9). Both effector-like loci (136/431; 36\%) and HGT genes $(38 / 151 ; 25 \%)$ were duplicated in the tandem duplications. Of effectors that 


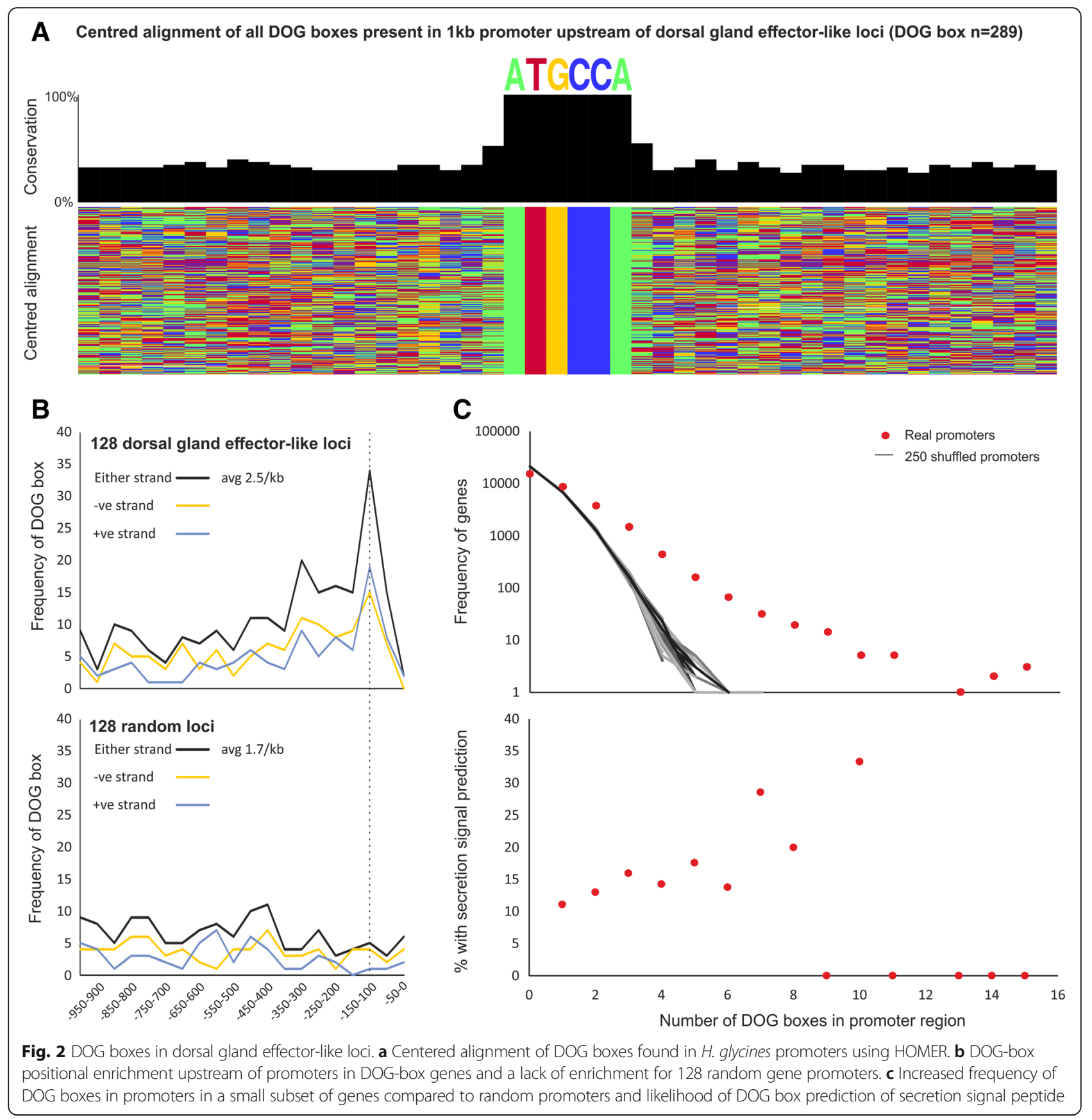

were orthologous in the tandemly duplicated orthologs, Hgg-20 (144), 4D06 (11) and 2D01 (11) were the most frequent, while RAN-binding proteins formed the largest cluster of HGT genes (Additional file 1: Figure S9).

To investigate whether transposons were associated with the expansion of effector genes, we created confident transposon and retrotransposon models using data co-integrated from RepeatModeler, LTR finder, and Inverted Repeat Finder (see methods). One-third of the H. glycines genome was considered repetitive by RepeatModeler (32\%, 39 Mb) with the largest classified types being DNA transposons
(7.53\%), LTR elements (2.92\%), LINEs (1.83\%) and SINEs (0.04\%) (Additional file 1: Table S4). To identify full-length DNA transposons and LTR retrotransposons, Inverted Repeat Finder (3.07) and LTR Finder (1.0.5) were used to identify terminal inverted repeats (TIRs) and LTRs, respectively. The genomic co-localization of RepeatModeler repeats and inverted repeats led to the identification of 1075 DNA transposons with a mean size of $6.6 \mathrm{~kb}$ and encompassing 1915 genes (Fig. 4). Similarly, the overlap of RepeatModeler repeats and LTR Finder repeats identified 592 LTR retrotransposons with $8.1 \mathrm{~kb}$ mean size and encompassing 


\section{A}

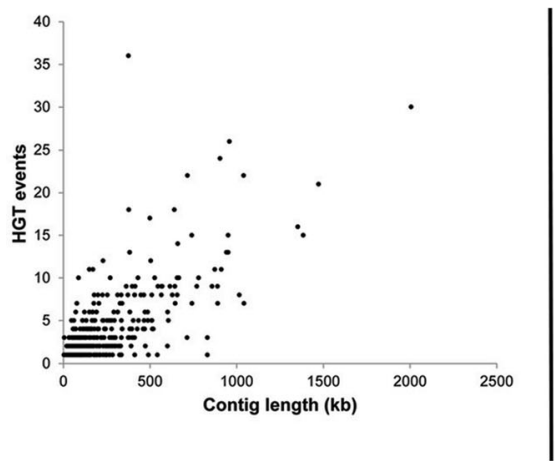

B

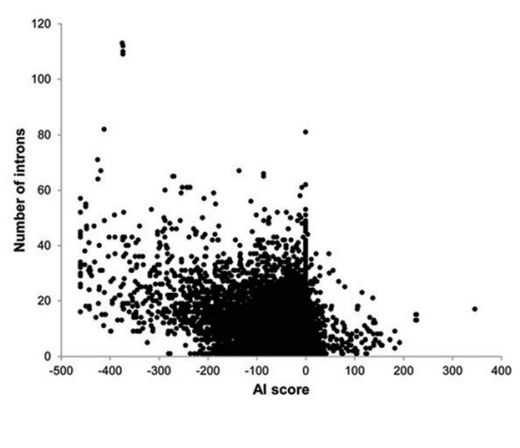

Fig. 3 SNP density (a) and expression (b) of effector genes and gene copy number variants. Effectors found within repetitive regions were not significantly associated with SNP changes, although both effectors and secreted genes in any region of the genome were frequently up and downregulated in expression. Significance was calculated with Fisher's exact tests in the GeneOverlap R package, and significance at $<0.05$ is *, $<0.01$ is ${ }^{* *}$. Expression is represented as a log fold change of expression in a comparison of pre-parasitic and parasitic $J 2$ nematodes

1401 genes (Fig. 4). Among the genes found within DNA or retro-transposon borders, 58/1915 and 22/1401 were predicted effectors, respectively. Indeed, many transposonassociated genes had effector-like functions (Additional file 1: Figure S9), as seen in a tandemly duplicated transposable element carrying known effectors and effector-like genes (Additional file 1: Figure S10). Transposon-mediated duplication is not specific to effectors, as evidenced by 14 duplicated HGT RAN-binding proteins. To obtain a measure of

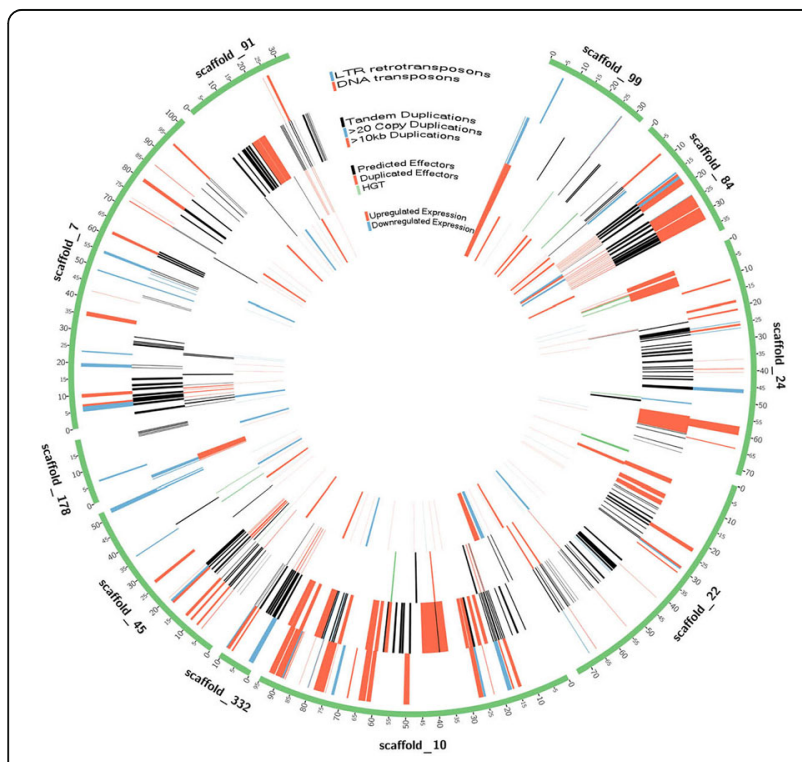

Fig. 4 Most highly duplicated scaffolds in the H. glycines genome colocalizing with effector genes. Scaffold ticks signify $50 \mathrm{~kb}$ increments along scaffolds (green), High correlations were found between regions containing transposon elements and tandem duplications. Effectors were frequently duplicated within these regions. HGT events were evenly distributed across scaffolds, while upregulated and downregulated expression was frequently associated with variable up and down regulation of expression duplications associated with transposons, Bedtools intersect was used to identify transposon-associated gene overlap with tandemly duplicated genes. Of the 6730 genes contained in tandem duplications, 969 and 656 were contained in DNA and LTR transposons, respectively.

Another possible mechanism by which $H$. glycines could overcome soybean resistance is through changes in coding sequences that result in differences among closely related effectors. Therefore, identifying SNPs in effector genes may reveal mutations associated with effector diversification. Using GATK best practices [31], 1,619,134 SNPs were identified from 15 bulked, pooled DNA preparations from isolate populations of virulent and avirulent $H$. glycines lines. To better understand population-level dynamics SNP-Relate was used to create a PCA plot, and as expected, populations primarily grouped by their original ancestral population but also by selection pressure on resistant cultivars (Additional file 1: Figure S11). The SNP density for each gene was determined by dividing SNP frequency by CDS length, and Fisher's exact tests with the GeneOverlap R package were used to identify significant associations with genes in the 10th and 90th percentile of SNP density (Fig. 5, Additional file 5: Table S5). SNP-dense genes were significantly enriched for genes found in tandem duplications, DNA transposons, LTR retrotransposons, and any gene with exon-overlapping repeats. While mutations are present in effectors, effector genes were not associated with high SNP density, although the lack of unique reads in highly duplicated regions may be responsible. Supporting this hypothesis, genes and effectors found in tandem duplications, DNA transposons, and LTR retrotransposons significantly overlapped with the 4613 genes lacking SNPs, and thus unique sequence reads (Fig. 5, Additional file 5: Table S5).

\section{Genomic structures associated with gene expression change in $\mathrm{H}$. glycines}

To assess the importance of genes affected by duplications, repeat-association, and SNP density, we utilized 


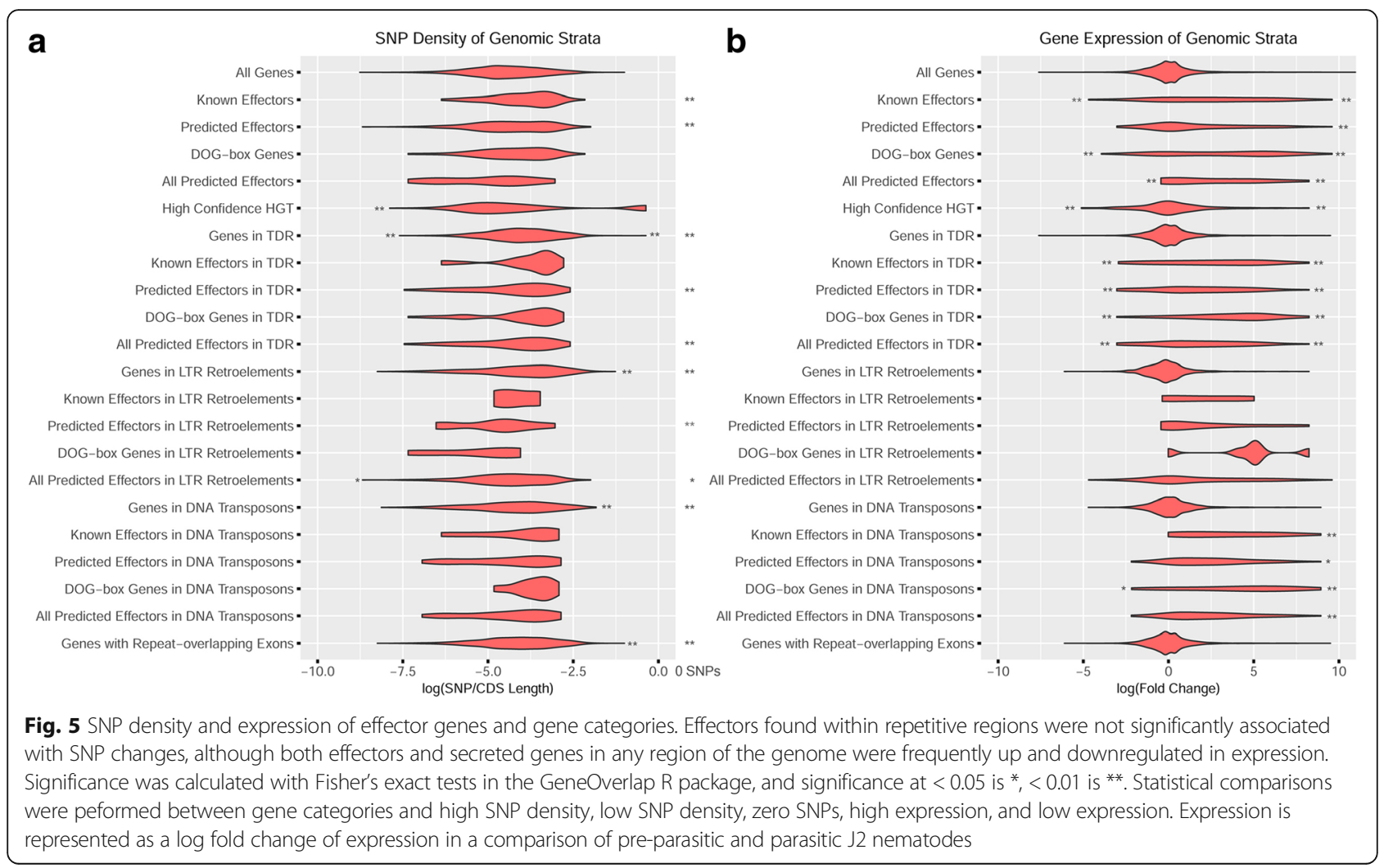

gene expression from second-stage juveniles of $\mathrm{H}$. glycines population PA3 before and after root infection of a resistant and susceptible soybean cultivar (SRP122521). Genes differentially up and downregulated after infection were identified using DESEQ with a q-value cutoff of 1e-8, revealing 1211 and 568 genes with significant up and down regulation, respectively. To associate differential expression with effectors and other gene categories, significant associations were identified using the GeneOverlap $R$ package (Fig. 5, Additional file 5: Table S5). As expected, many of the predicted effectors were significantly upregulated upon infection, a trend that continued with putative effectors found in DNA transposons and tandem duplications. In contrast, the only significantly upregulated gene categories not directly associated with predicted effectors were secreted genes and genes associated with an effector-associated repeat (Family-976 repeat).

However, since virulence genes have a limited span of use before host immunity is developed, the expression of a recognized effector may hinder survival, thus finding effectors with reduced gene expression is not surprising. Generally, genes associated with tandem duplications, HGT, and transposons had similar distributions of expression as genes that were non-associated, yet effectors found in tandem duplications and DNA transposons were significantly enriched for genes with high and low expression (Fig. 5). This high and low expression trend in effectors was also apparent in secreted genes at a higher significance, indicating that many potential effectors remain elusive to detection.

\section{Discussion}

To overcome the expected assembly problems associated with high-levels of repetitive DNA and to reveal the evolutionary means behind the rapid evolution and population shifts in $H$. glycines, we used long-read technology to assemble a genome from a heterogenous population of individuals. Several analyses confirmed a high level of genome completeness with $\sim 88 \%$ of the RNA-Seq aligning, 93\% of preads aligning, and zero contaminating scaffolds (Additional file 1: Table S1, Figure S1). While percentages of missing BUSCO [32] genes were high, BUSCO genes were $72 \%$ complete, ranking $H$. glycines the best among sequenced genomes in the cyst and knot-nematode clades (Fig. 1, Additional file 1: Table S2). Some level of artifactual duplication may be present in the genome, with BUSCO gene duplication being highest among the species analyzed. However, only 79/ 349 duplicated BUSCO genes are found in tandem duplications, indicating that duplication or heterozygous contigs may be present elsewhere in the genome. With a goal-oriented approach of capturing all genic variation in the genome, we sequenced a population of multiple 
individuals. We therefore assembled a chimera of individuals, with some duplicated genes originating from single variants in the population. However, even when considering that nearly nine thousand genes could be attributed to repetitive elements and tandem duplications, the gene frequency $(20,830)$ and exon statistics of $H$. glycines are elevated in relation to sister Tylenchida species.

Because plant parasitism has independently arisen three times in Nematoda, and because it is thought that HGT plays a crucial role in the nematodes' adaptation to this lifestyle [25, 33], we investigated the potential role HGT may have in $H$. glycines. Almost all previously identified HGT in plant-parasitic nematodes were also found in $H$. glycines $(n=82)$ (Additional file 1: Table S3) [34]. Genes with strong AI ( $>30$ ) were mainly hydrolases, transferases, oxidoreductases or transporters (Additional file 3: Data S2). Of interest were genes originating from bacteria or fungi, but lacking BLAST hits to Metazoan species (highlighted blue in Additional file 4: Data S3). Among these is a gene coding for an Inosine-uridine preferring nucleoside hydrolase (Hetgly.000009703; $\mathrm{AI}=101.2$ ), an enzyme essential for parasitism in many plant-pathogenic bacteria and trypanosomes [35]. A candidate oomycete RxLR effector [36] was also identified in the genome (Hetgly.000002962, Hetgly.000002964 and Hetgly.000002966; AI up to 42.2). Besides being necessary for successful infection, RxLR effectors are also avirulence genes in some species, including the soybean pathogen Phytophthora sojae [37]. The H. glycines genome is also host to a putative HGT gene (Hetgly.000001822 and Hetgly.000022293; AI up to 55.3) that has been characterized as $a$ G. pallida effector (Gp-FAR-1) involved in plant defense evasion by binding plant defense compounds [38]. Thus, horizontal gene transfer appears to contribute to the evolution of $H$. glycines virulence as well as to the ancestral development of parasitism in plant-parasitic nematodes [33, 39-41].

Although HGT is more common among nematodes and arthropods than other animals [42], there are many documented cases of gene duplication leading to evolutionary novelty and phenotypic adaptation across metazoans $[43,44]$. With over a fifth of the genes in the $H$. glycines genome found in tandem duplications, characterizing the largest clusters of orthologous gene families in tandem duplications provides relevant information for identifying genes related to parasitism, adaptation, and virulence. A functional assessment of the 38 largest clusters of tandemly duplicated orthologues were largely transposon-associated proteins or proteins related to effectors, indicating that transposons have a role in duplicating effector genes (Additional file 1: Figures S9, S10). Because many of the LTRs and TIRs were nested, the frequent rearrangements of nested clusters of transposons [45] could be attributed to effector exon shuffling
[46]. While genes in duplicated regions of the genome were significantly associated with high SNP density (Fig. 5a), putative effectors were not. While it is known that genes in duplicated regions pave a way for evolutionary novelty $[43,44]$, the lack of high SNP density for effectors in duplicated regions may represent low sequencing depth or the recent duplication of these loci. While significant effector mutations could not be found in these regions, these effectors were some of the most highly upregulated and downregulated genes upon infection (Fig. 5b).

\section{Conclusions}

The H. glycines genome assembly and annotation provides a glimpse into host and parasite interplay through the characterization of known and predicted effector genes. This relationship is further unraveled through the characterization of tandem duplications, horizontal gene transfers, transposon hitchhiking, promotor regulatory element identification, alternative splicing, SNP density, and gene expression. The generation of these genomic resources will facilitate a greater understanding of the host-parasite relationship by revealing genes involved in creating and maintaining a functional feeding site. Thus, the genomic analysis of the $H$. glycines genome is an important advance in the pathway to generating new forms of resistance and control measures against $H$. glycines.

\section{Methods}

\section{Nematode culture and DNA/RNA isolation}

H. glycines inbred population TN10, Hg type 1.2.6.7, was grown on susceptible soybean cultivar Williams 82 in a greenhouse at Iowa State University. A starting culture of approximately 10,000 eggs from Dr. Kris Lambert, University of Illinois, was bulked for four generations on Williams 82 soybeans grown in a 2:1 mixture of steam pasteurized sand:field soil in 8 " clay pots, with approximately $16 \mathrm{~h}$ daylight at $27^{\circ} \mathrm{C}$. Genomic DNA was extracted from approximately 100,000 eggs in a subset of third generation cysts. Egg extraction was performed with standard nematological protocols [47], eggs washed 3 times in sterile $10 \mathrm{mM}$ MES buffered water, and pelleted before flash freezing in liquid nitrogen.

Genomic DNA was isolated using the MasterPure Complete DNA Purification Kit (Epicentre) with the following modifications: Frozen nematode eggs were resuspended in $300 \mathrm{ul}$ of tissue and cell lysis solution, and immediately placed in a small precooled mortar, where the nematode solution refroze and was finely ground. The mortar was then placed in a $50{ }^{\circ} \mathrm{C}$-water bath for $30 \mathrm{~min}$, then transferred to $500 \mathrm{ul} \mathrm{PCR}$ tubes with $1 \mathrm{ul}$ of proteinase $\mathrm{K}$, and incubated at $65^{\circ} \mathrm{C}$ for $15 \mathrm{~min}$, inverting every $5 \mathrm{~min}$. Genomic DNA was resuspended in $30 \mathrm{ul}$ of RNAse/DNase free water, quantified via 
nanodrop, and inspected with an $0.8 \%$ agarose gel at 40 $\mathrm{V}$ for $1 \mathrm{~h}$. Two $20 \mathrm{~kb}$ insert libraries were generated and sequenced on 20 PacBio flow cells at the National Center for Genome Resources in Santa Fe, NM (SRR5397387 - SRR5397406).

Fifteen H. glycines populations were chosen based on $\mathrm{Hg}$-type diversity and were biotyped to ensure identity (TN22, TN8, TN7, TN15, TN1, TN21, TN19, LY1, OP50, OP20, OP25, TN16, PA3, G3). Information on the selection and Hg-types of these lines is available in Additional file 6: Table S6. Genomic DNA from approximately 100,000 eggs for each population was extracted as described previously, and $500 \mathrm{bp}$ libraries were sequenced on an Illumina HiSeq 2500 at 100PE (SRR5422809 - SRR5422824).

Six life stages were isolated for both PA3 and TN19 $H$. glycines populations: eggs, pre-parasitic second-stage juveniles (J2), parasitic J2, third-stage juveniles (J3), fourth stage juveniles (J4) and adult females. Parasitic J2 were isolated, followed by isolations of J3, J4, and adult females at $3,8,15$, and 24 days post-infection via a combination of root maceration, sieving and sucrose floatation, using standard nematological methods [47]. Total RNA was extracted with the Exiqon miRCURY RNA Isolation Kit (Catalog \#300112). RNA was combined to form three pools for each population, corresponding to early (egg and pre-parasitic J2), middle (parasitic J2 and J3) and late (J4 females and early adult females) developmental stages. The IsoSeq data were used to improve the annotation (see below) (SAMN08541516-SAMN08541521).

\section{Genome assembly}

A PacBio subreads assembly was generated with Falcon to correct subreads into consensus preads (error corrected reads), followed by contig assembly. An alternative approach using only transcript containing preads was helpful in solving heterozygosity and population problems. Transcripts were aligned to preads using Gmap [48] under default parameters, and a pool of preads for each unique transcript was assembled using CAP3 [49] under default parameters. The longest assembled contigs and all unassembled preads were retained and read/contig redundancy was removed with sort and uniq. New FASTA headers were generated using nanocorrect-preprocess.pl [https://github.com/jts/nanocorrect/blob/master/nanocorrect-preprocess.pl], and sequences were then assembled with Falcon with default settings into 2692 contigs (supp file $H$. glycines.cfg). Falcon output was converted to Fastg with Falcon2Fastg [https://github.com/md5sam/Falcon2Fastg], and longer scaffolds were created with Bandage [50] using multiple criteria. 1) The longest path was chosen and ended with an absence of edges. 2) If the orientation of an interior contig was disputed, one set of edges was deleted to extend the scaffold. 3) The shortest path through difficult repetitive subgraphs was chosen.

Intragenomic synteny was used to remove clonal haplotigs $[51,52]$ (synteny as below). When synteny was identified between two contigs/scaffolds, if a longer 3' or $5^{\prime}$ fragment could be made, then the ends of each contig/scaffold were exchanged at the syntenic/non-syntenic juncture. All remaining duplicate scaffolds retaining synteny were truncated or removed from the assembly, and followed by a BWA [53] self-alignment to remove redundant repetitive scaffolds at a $90 \%$ identity threshold.

\section{Genome quality control}

Multiple measures were taken to assess genome assembly quality, including a default BLASR [54] alignment of PacBio subreads, preads, and ccsreads resulting in alignment percentages at 88.7, 93.3, 90.1\%, respectively (Additional file 1: Table S1). Using default settings, Gmap and Hisat2 (2.0.3) mapped $86.4 \%$ percent of a transcriptome assembly and $\sim 88 \%$ of the five RNA-seq libraries, respectively (Additional file 1: Table S1). Genome completeness was assessed with BUSCO [32] at 71.9\%. An absence of contamination was found with Blobtools (4.8.2) [55] using MegaBLAST (2.2.30+) to the NCBI nt database, accessed 02/02/17, at a 1-e5 e-value. See Additional file 7 for more detail.

\section{Genome annotation}

To account for the high proportion of noncanonical splicing in nematodes [12], Braker [56] was used to predict genes using Hisat2 (2.0.3) [57] raw RNA-Seq alignments of $\sim 230$ million $100 \mathrm{bp} \mathrm{PE} \mathrm{RNA-Seq} \mathrm{reads} \mathrm{[14]} \mathrm{and}$ GMAP [58] alignments of IsoSeq reads, and all EST sequences from NCBI. Because gene models were greatly influenced by repeat masking, three differentially repeat-masked genomes were used for gene prediction: unmasked, all masked, and all except simple repeats masked (see supp table RNASEQ mapping in excel). All protein isoforms were annotated with Interproscan [59, 60] in BlastGO [61], and with BLAST [62] to Swiss-prot [63] and Uniref [64] at e-value 1e-5.

\section{Repeat prediction}

Repetitive elements in the $H$. glycines genome were classified into families with five rounds of RepeatModeler (1.0.8) [65] at default settings, followed by genome masking with RepeatMasker [66] at default settings. Inverted Repeat Finder (3.07) and LTR Finder (1.0.5) were used at default settings to define the border of a TE only when overlapping RepeatModeler repeats were 
present. Supplemental helitron prediction was done with HelitronScanner [67] under default settings.

\section{Promoter analyses}

To determine to what extent cyst nematodes use common mechanisms for dorsal gland effector regulation, we screened sequences previously associated with the DOG box in other genera against the $H$. glycines genome. The G. rostochiensis DOG-effectors [12] were used as queries in BLASTp to identify DOG-effector-like loci in the predicted proteome of $\mathrm{H}$. glycines. The most similar sequence was retrieved if it satisfied two criteria, an e-value $<1 \mathrm{e}-10$ and the protein encoded a signal peptide for secretion (78 unique $H$. glycines loci). Using the same approach, 94 genes similar to other published dorsal gland expressed effectors (58) were identified [6] and combined with the DOG-effector-like list to a non-redundant 128 loci. Given the nature of these two criteria, not all sequences in this list will be effectors and not all effectors will be in this list, nevertheless, it will contain a sufficient number to determine whether the DOG box is conserved in $H$. glycines. A 500 bp region 5' of the ATG start codon, termed the promoter region, was extracted from these 128 loci and used for motif enrichment analysis using HOMER [68], as previously described [12]. DOG-box positional enrichment was calculated using FIMO web server [69] and predictive power calculated using custom python scripts.

\section{Effector prediction}

At default settings Gmap [58] was used to align 80 previously identified effectors to the genome $[5,6,70,71]$. Conserved protein motifs in effectors were identified with MEME: -nmotifs 24,-minsites 5, -minw 7, -maxw 300 , and zoops (zero or one per sequence) [72]. These motifs were used as FIMO queries to search the inferred H. glycines proteome [72].

\section{Synteny}

The genome, gff, and peptide sequences for C. elegans (WBcel235), G. pallida [73], and M. hapla [74] were downloaded from WormBase [75]. The genome and gff of G. rostochiensis [12] was downloaded from NCBI. The G. ellingtonae genome was also downloaded from NCBI [76], but gene models were unavailable, thus gene models for G. ellingtonae were called with Braker using RNA-seq reads from SRR3162514, as described earlier.

Fastp and global alignments with Opscan (0.1) [77] were used to calculate orthologous gene families between $H$. glycines and C. elegans [78], G. pallida [73], G. ellingtonae [76], G. rostochiensis [12], M. hapla [79], and M. incognita [15]. All alternatively spliced variants and all possible multi-family genes were considered $(-\mathrm{C},-\mathrm{b},-\mathrm{Q})$.
To infer synteny, iAdHoRe 3.0.01 [80] was used with prob_cutoff $=0.001$, level 2 multiplicons only, gap_size $=$ 15 , cluster_gap $=20$, q_value $=0.9$, and a minimum of 3 anchor points. Syntenic regions are displayed using Cir$\cos (0.69 .2)$ [81].

\section{Phylogenetics}

Predicted protein sequences from the aforementioned nematode genomes (excluding C. elegans) were scanned with BUSCO 2.0 [32] for 982 proteins conserved in nematoda_odb9. 651 proteins were found in at least 3 species and aligned with Prank [82] in Guidance [83] at default parameters. Maximum likelihood gene trees were computed using RAxML [84] with 1000 bootstraps and PROTGAMMAAUTO for model selection. Astral [85] at default settings was used to prepare a coalescent-based species tree.

\section{Tandem duplication}

With default settings, ReDtandem.pl was used to identify tandem duplications in the genome [86]. Tandem duplicate orthologous genes were identified using a self-BLASTp to predicted proteins with 50\% query length and $90 \%$ identity [62]. To annotate clusters of orthologous genes, groups of highly connected nodes or entire clusters were concatenated and queried with BLASTp to the NCBI NR database [87].

\section{SNP density and PCA analysis of fifteen $H$. glycines populations}

Raw sequences from fifteen populations of $H$. glycines nematodes were quality checked with FastQC [88]. Virulence for each $\mathrm{H}$. glycines population are available in Additional file 6: Table S6. Reads were aligned to the $H$. glycines genome using default parameters in BWA-MEM [53]. The BAM files were sorted, cleaned, marked for duplicates, read groups were added and SNP/Indel realignment were performed prior to calling SNPs and Indels with GATK. Custom Bash scripts were used to convert the vcf file into a gff for use with Bedtools (2.2.6) to identify SNP and exon overlap [89]. The density of SNPs was calculated by dividing the number of SNPs/CDS length (bp). Phasing and imputing SNPs with Beagle $4.1[90,91]$ followed by a PCA analysis of SNPs vs Hg-type virulence using SNPRelate (1.12.2) [92].

\section{RNA-seq expression}

RNA-seq reads were obtained from NCBI SRA accession SRP122521. Briefly, SCN inbred population PA3 was grown on soybean cultivar Williams 82 or EXF63. Pre-parasitic second-stage juveniles and parasitic second stage juveniles were isolated from roots of resistant and susceptible cultivars at 5 days post-inoculation [14]. 100 bp PE reads were aligned to the genome using default 
settings with HiSat2 [57]. Read counts were calculated using default settings with FeatureCounts from the Subread package [93], followed by Deseq2 [94] at default settings to determine log-fold change between the pre-parasitic samples $(2 \times$ ppJ2_PA3 $)$ and parasitic J2 samples $(2 \times$ pJ2_s63, pJ2_race3_Forrest).

\section{Alternative splicing}

The analysis of the global changes and effector specific effects in alternative splicing landscape was assessed following a recent de novo transcriptomics analysis of the H. glycines nematode effectors [14] Transcriptome annotation was constructed using 230 million RNA-Seq reads from both pre-parasitic and parasitic J2 H. glycines [14], 34,041 iso-seq reads from three life stages of both a virulent and an avirulent strain, and $H$. glycines ESTs in NCBI $(35,796)$. Specifically, using a standard alternative splicing analysis pipeline [95], 230 million reads from both pre-parasitic and parasitic J2 H. glycines [14] were preprocessed with Trimmomatic [96], aligned with Tophat 2.1.1 [97], and quantified with Cufflinks 2.2.1 [98], followed by conversion of FPKM to TPM [99], and patterns assessment with IsoformSwitchAnalyzerR [100]. For the 80 previously identified effectors $[6,70,71]$, the changes in the functional domain architectures between specific alternatively spliced isoforms are determined using InterPro domain annotation server with a focus on Pfam domains [101].

\section{Additional files}

Additional file 1: Figure S1. Contamination check with Blobtools. Circles represent scaffolds, while their colors represent different Phyla. All putative contaminating scaffolds are false-positive and have $\mathrm{H}$. glycines origins. The one outlier represents the mitochondrial scaffold, which was misassembled and collapsed to appropriate size. Table S1. Rates of read alignment to the genome for PacBio reads, RNA-seq, and Trinity transcripts. Table S2. Busco genes found in Complete, Single-copy, Duplicated, Fragmented, and Missing categories for assembled genomes in the Tylenchida. Figure S2. Globodera rostchiensis synteny. 439 syntenic regions were identified between $\mathrm{G}$. rostochiensis and H. glycines. Green contigs are $\mathrm{H}$. glycines, while blue contigs are G. rostochiensis. Figure S3. Globodera pallida synteny. 341 syntenic regions were identified between G. pallida and H. glycines. Green contigs are H. glycines, while blue contigs are G. pallida. Figure S4. Globodera ellingtonae synteny. 362 syntenic regions were identified between G. ellingtonae and H. glycines. Green contigs are $\mathrm{H}$. glycines, while blue contigs are G. ellingtonae. Figure S5. Meloidogyne hapla synteny. 112 syntenic regions were identified between M. hapla and H. glycines. Green contigs are H. glycines, while blue contigs are M. hapla. Figure S6. Meloidogyne incognita synteny. 15 syntenic regions were identified between $M$. incognita and H. glycines. Green contigs are H. glycines, while blue contigs are $\mathbf{M}$. incognita. Figure S7. Repeatmodeler contig alignments overlapping effector alignments in the genome. Three separate examples, with the top track representing final gene models, middle representing Repeatmodeler/Repeatmasker contig alignments, and the lower track representing known effector alignments. Figure S8. Motif analysis of effector sequences. The 80 known effector proteins were subjected to a MEME analysis, and motifs identified in 61 effector proteins implemented with FIMO to find effector candidates in the genome. Table S3. H. glycines genes previously shown to be acquired by horizontal gene transfer in closely related plant-parasitic nematodes. Figure S9. Network of interrelated tandemly duplicated genes. Connections indicate protein similarity, while the text represents the BLAST hits to NR for the three most highly connected nodes in each subnetwork (hexagons). Table S4. Repeatmodeler/Repeatmasker repeats identified in the H. glycines genome. Figure S10. Colocalized transposons, tandem duplications, and effectors. A. JBrowse display of scaffold_345, showing four tracks: Gene annotations, DNA transposons, 80 known effector alignments, and tandem duplications. The large transposon colocalizes with five tandem duplications, four of the 80 known effectors, and four genes annotated as effectors. B. A highly similar transposon on scaffold_97 with the same effector types present within and tandem duplications nearby. Figure S11. Principal components analysis of SNPs from 15 populations of $\mathrm{H}$. glycines nematodes. Colors represent Hg-type, the capability to reproduce to a certain threshold on seven soybean cultivars. The labels by each circle represent the names of each population. Figure S12. Alternative splicing changes in isoforms for all genes in the genome. All isoforms containing an ORF in the transcriptome were analyzed for three biological groups, and all pairwise comparisons were considered to show the changes in transcript structures caused by alternative splicing. AS isoform structures are characterized by three types of annotations: intron retention, NMD (nonmediated decay), and effect on ORF. Figure S13. Alternative splicing changes in isoforms for all effectors in the genome. Effector isoforms containing an ORF in the transcriptome were analyzed for three biological groups, and all pairwise comparisons were considered to show the changes in transcript structures caused by alternative splicing. AS isoform structures are characterized by three types of annotations: intron retention, NMD (nonmediated decay), and effect on ORF. (DOCX $6288 \mathrm{~kb}$ )

Additional file 2: Data S1. MEME motifs annotated with BLAST annotations to NR. Annotated MEME motifs from effector motif-finding. (TXT $1 \mathrm{~kb}$ )

Additional file 3: Data S2. All horizontal gene transfer events. All putative horizontal gene transfer events above an alien index of zero. (XLSX $398 \mathrm{~kb})$

Additional file 4: Data S3. Horizontal gene transfer events novel to $H$. glycines. Horizontal gene transfer events not previously reported in other plant parasitic nematodes. (XLSX $19 \mathrm{~kb}$ )

Additional file 5: Table S5. Significance tests for gene expression and snp density (XLSX $15 \mathrm{~kb}$ )

Additional file 6: Table S6. Nematode isotypes and selection. Hg-types, selection, and heritage of nematodes using in sequencing. (XLSX $12 \mathrm{~kb}$ )

Additional file 7: Supporting analyses text. (DOCX $22 \mathrm{~kb}$ )

\section{Abbreviations}

Al: Alien index; BTB: BR-C, ttk, and bab domain containing; CDS: Coding region; DNA: Deoxyribonucleic acid; DOG: Dorsal expressed gene; EST: Expressed sequence tag; GS-like: Glutathione synthase-like; HGT: Horizontal gene transfer; LINE: Long interspersed nuclear element; LTR: Long terminal repeat; NR: Non-redundant protein database; PCA: Principal components analysis; POZ: Pox virus and zinc finger domain; RAN: RAs-related Nuclear protein; RNA: Ribonucleic acid; SCN: Soybean cyst nematode; SINE: Short interspersed nuclear element; SNP: Single nucleotide polymorphism; SPRY-SEC: Secreted SPRY domain-containing protein;

TD: Tandem duplication; TIR: Terminal inverted repeat.

\section{Acknowledgements}

We thank the National Center for Genome Resources in Santa Fe, NM for performing PacBio sequencing, and lowa State DNA Facility in Ames, IA for Illumina sequencing of fifteen populations. We also thank Levi Baber for IT support in visualizing genomics data with JBrowse.

\section{Funding}

RM, TRM, PSJ, MGM, MH, AJS and TJB would like to acknowledge the critical support of the North Central Soybean Research Program. Work conducted by the U.S. Department of Energy Joint Genome Institute is supported by 
the Office of Science of the U.S. Department of Energy under Contract No. DE-AC02-05CH1 1231. SEvdA is supported by Biotechnology and Biological Sciences Research Council grant BB/R011311/1. DK and NTJ acknowledge support by National Science Foundation (DBI-1458267 to DK). This work used the Extreme Science and Engineering Discovery Environment (XSEDE) [102], which is supported by National Science Foundation grant number ACl1548562. Specifically, it used the Bridges system [103], which is supported by NSF award number ACl-1445606, at the Pittsburgh Supercomputing Center (PSC). BM and EL are supported by Genome Canada, Genome Quebec and partners listed on soyagen.ca. PacBio sequencing was obtained using funds from the National Science Foundation I/UCRC, the Center for Arthropod Management Technologies under Grant No. IIP-1338775 and by industry partners.

\section{Availability of data and materials}

Datasets generated during the current study are available at Genbank accessions (SRR6782833 - SRR6782842), (SRR5397387 - SRR5397406), (SRR5422809 - SRR5422824). BioProject address: https:// www.ncbi.nlm.nih.gov/bioproject/?term=PRJNA381081. Scripts used for the alternative splicing analysis can be found at https://github.com/bioinfonerd/ SCN_AS_RNA_Seq. Scripts used for the promoter analysis can be found here: https://github.com/sebastianevda/Fimo_parse/tree/master. All other scripts and bioinformatic analyses can be found at: https://github.com/ remkv6/SCN_Genome_Paper.

\section{Authors' contributions}

RM, TRM, PSJ, MGM, MH, AJS, UM, JS, AS, and TJB conceived and designed the experiment. TRM isolated and acquired the data. RM and AJS performed the assembly. SEvdA performed and wrote the promoter analysis. DK and NTJ performed and wrote the alternative splicing analysis. BM and EL performed and wrote the horizontal gene transfer analysis. RM performed all other comparative analyses. All authors made substantial contributions to the final text. All authors read and approved the final manuscript.

\section{Ethics approval and consent to participate}

Not applicable.

\section{Consent for publication}

Not applicable.

\section{Competing interests}

The authors declare that they have no competing interests.

\section{Publisher's Note}

Springer Nature remains neutral with regard to jurisdictional claims in published maps and institutional affiliations.

\section{Author details \\ ${ }^{1}$ Department of Plant Pathology, lowa State University, Ames, IA, USA. ${ }^{2}$ Genome Informatics Facility, lowa State University, Ames, IA, USA. ${ }^{3}$ Agriculture and Agri-Food Canada, Saint-Jean-sur-Richelieu, QC, Canada. ${ }^{4}$ Department of Energy, Joint Genome Institute, Walnut Creek, CA, USA. ${ }^{5}$ HudsonAlpha Institute for Biotechnology, Huntsville, AL, USA. ${ }^{6}$ Bioinformatics and Computational Biology Program, Worcester Polytechnic Institute, Worcester, MA, USA. 'Department of Computer Science, Worcester Polytechnic Institute, Worcester, MA, USA. ${ }^{8}$ Division of Plant Sciences, University of Missouri, Columbia, MO, USA. ${ }^{9}$ Department of Plant Sciences, University of Cambridge, Cambridge, UK. ${ }^{10}$ Department of Crop Sciences University of Illinois, Urbana, IL, USA.}

\section{Received: 28 September 2018 Accepted: 28 January 2019} Published online: 07 February 2019

\section{References}

1. Koenning SR, Wrather JA. Suppression of soybean yield potential in the continental United States from plant diseases estimated from 2006 to 2009. Plant Health Prog. 2010. https://doi.org/10.1094/PHP-2010-1122-01-RS.

2. Niblack T, Lambert K, Tylka G. A model plant pathogen from the kingdom animalia: Heterodera glycines, the soybean cyst nematode. Annu Rev Phytopathol. 2006;44:283-303.
3. Endo BY. Penetration and development of Heterodera glycines in soybean roots and related anatomical changes. Phytopath. 1964;54:79-88.

4. Hussey RS. Disease-inducing secretions of plant-parasitic nematodes. Annu Rev Phytopathol. 1989;27(1):123-41.

5. Gao B, Allen R, Maier T, Davis EL, Baum TJ, Hussey RS. The parasitome of the phytonematode Heterodera glycines. Mol Plant-Microbe Interact. 2003;16(8):720-6.

6. Noon JB, Hewezi TAF, Maier TR, Simmons C, Wei J-Z, Wu G, Llaca V, Deschamps S, Davis E, Mitchum M. Eighteen new candidate effectors of the phytonematode Heterodera glycines produced specifically in the secretory esophageal gland cells during parasitism. Phytopathology. 2015; (ja).

7. Hewezi T, Baum TJ. Manipulation of plant cells by cyst and root-knot nematode effectors. Mol Plant-Microbe Interact. 2013;26(1):9-16.

8. Hewezi T. Cellular signaling pathways and posttranslational modifications mediated by nematode effector proteins. Plant Physiol. 2015;169(2):1018-26.

9. Juvale PS, Baum TJ: "Cyst-ained" research into Heterodera parasitism. PLoS Pathog 2018, 14(2):e1006791.

10. Mitchum MG, Hussey RS, Baum TJ, Wang X, Elling AA, Wubben M, Davis EL. Nematode effector proteins: an emerging paradigm of parasitism. New Phytol. 2013;199(4):879-94.

11. Tylka GL. Understanding soybean cyst nematode HG types and races. Plant Health Progress. 2016;17(2):149.

12. Eves-van den Akker S, Laetsch DR, Thorpe P, Lilley CJ, Danchin EG, Da Rocha M, Rancurel C, Holroyd NE, Cotton JA, Szitenberg A. The genome of the yellow potato cyst nematode, Globodera rostochiensis, reveals insights into the basis of parasitism and virulence. Genome Biol. 2016;17(1):124.

13. Lapp N, Triantaphyllou A. Relative DNA content and chromosomal relationships of some Meloidogyne, Heterodera, and Meloidodera spp. (Nematoda: Heteroderidae). J Nematol. 1972;4(4):287.

14. Gardner M, Dhroso A, Johnson N, Davis EL, Baum TJ, Korkin D, Mitchum MG. Novel global effector mining from the transcriptome of early life stages of the soybean cyst nematode Heterodera glycines. Sci Rep. 2018;8(1):2505.

15. Abad P, Gouzy J, Aury J-M, Castagnone-Sereno P, Danchin EG, Deleury E, Perfus-Barbeoch L, Anthouard V, Artiguenave F, Blok VC. Genome sequence of the metazoan plant-parasitic nematode Meloidogyne incognita. Nat Biotechnol. 2008:26(8):909.

16. Triantaphyllou A. An advance treatise on Meloidogyne vol. 1. Raleigh, USA: North Carolina State University Graphics; 1985.

17. Castagnone-Sereno P. Genetic variability and adaptive evolution in parthenogenetic root-knot nematodes. Heredity. 2006;96(4):282-9.

18. Lilley CJ, Maqbool A, Wu D, Yusup HB, Jones LM, Birch PR, Banfield MJ, Urwin PE, Eves-van den Akker S. Effector gene birth in plant parasitic nematodes: Neofunctionalization of a housekeeping glutathione synthetase gene. PLoS Genet. 2018;14(4):e1007310.

19. Nielsen H. Predicting secretory proteins with SignalP. In: Protein Function Prediction: Methods and Protocols; 2017. p. 59-73.

20. Mei Y, Thorpe P, Guzha A, Haegeman A, Blok VC, MacKenzie K, Gheysen G, Jones JT, Mantelin S. Only a small subset of the SPRY domain gene family in Globodera pallida is likely to encode effectors, two of which suppress host defences induced by the potato resistance gene Gpa2. Nematology. 2015; 17(4):409-24.

21. Scholl EH, Thorne JL, McCarter JP, Bird DM. Horizontally transferred genes in plant-parasitic nematodes: a high-throughput genomic approach. Genome Biol. 2003;4(6):R39.

22. Danchin EG, Rosso M-N, Vieira P, de Almeida-Engler J, Coutinho PM, Henrissat B, Abad P. Multiple lateral gene transfers and duplications have promoted plant parasitism ability in nematodes. Proc Natl Acad Sci. 2010; 107(41):17651-6.

23. Jones JT, Furlanetto C, Kikuchi T. Horizontal gene transfer from bacteria and fungi as a driving force in the evolution of plant parasitism in nematodes. Nematology. 2005;7(5):641-6.

24. Bird DM, Koltai H. Plant parasitic nematodes: habitats, hormones, and horizontally-acquired genes. J Plant Growth Regul. 2000;19(2):183-94.

25. Haegeman A, Jones JT, Danchin EG. Horizontal gene transfer in nematodes: a catalyst for plant parasitism? Mol Plant-Microbe Interact. 2011;24(8):879-87.

26. Mitreva M, Smant G, Helder J. Role of horizontal gene transfer in the evolution of plant parasitism among nematodes. Horizontal Gene Transfer. Humana Press; 2009. p. 517-535.

27. Smant G, Stokkermans JP, Yan Y, De Boer JM, Baum TJ, Wang X, Hussey RS, Gommers FJ, Henrissat B, Davis EL. Endogenous cellulases in animals: 
isolation of $\beta-1$, 4-endoglucanase genes from two species of plant-parasitic cyst nematodes. Proc Natl Acad Sci. 1998;95(9):4906-11.

28. Noon JB, Baum TJ. Horizontal gene transfer of acetyltransferases, invertases and chorismate mutases from different bacteria to diverse recipients. BMC Evol Biol. 2016;16(1):74.

29. Gladyshev EA, Meselson M, Arkhipova IR. Massive horizontal gene transfer in bdelloid rotifers. Science. 2008;320(5880):1210-3.

30. Young ND. The genetic architecture of resistance. Curr Opin Plant Biol. 2000; 3(4):285-90

31. DePristo MA, Banks E, Poplin R, Garimella KV, Maguire JR, Hartl C, Philippakis AA, Del Angel G, Rivas MA, Hanna M. A framework for variation discovery and genotyping using next-generation DNA sequencing data. Nat Genet. 2011;43(5):491.

32. Simão FA, Waterhouse RM, loannidis $P$, Kriventseva EV, Zdobnov EM. BUSCO: assessing genome assembly and annotation completeness with single-copy orthologs. Bioinformatics. 2015;31(19):3210-2.

33. Danchin EG, Guzeeva EA, Mantelin S, Berepiki A, Jones JT. Horizontal gene transfer from bacteria has enabled the plant-parasitic nematode Globodera pallida to feed on host-derived sucrose. Mol Biol Evol. 2016;33(6):1571-9.

34. Craig JP, Bekal S, Hudson M, Domier L, Niblack T, Lambert KN. Analysis of a horizontally transferred pathway involved in vitamin B6 biosynthesis from the soybean cyst nematode Heterodera glycines. Mol Biol Evol. 2008;25(10): 2085-98.

35. Gopaul DN, Meyer SL, Degano M, Sacchettini JC, Schramm VL. Inosineuridine nucleoside hydrolase from crithidia fasciculata. Genetic characterization, crystallization, and identification of histidine 241 as a catalytic site residue. Biochemistry. 1996;35(19):5963-70.

36. Morgan W, Kamoun S. RXLR effectors of plant pathogenic oomycetes. Curr Opin Microbiol. 2007;10(4):332-8.

37. Shan W, Cao M, Leung D, Tyler BM. The Avr1b locus of Phytophthora sojae encodes an elicitor and a regulator required for avirulence on soybean plants carrying resistance gene Rps 1b. Mol Plant-Microbe Interact. 2004 17(4):394-403.

38. Prior A, Jones JT, Blok VC, Beauchamp J, McDermott L, Cooper A, Kennedy MW. A surface-associated retinol-and fatty acid-binding protein (Gp-FAR-1) from the potato cyst nematode Globodera pallida: lipid binding activities, structural analysis and expression pattern. Biochem J. 2001;356(Pt 2):387.

39. Danchin EG, Perfus-Barbeoch L, Rancurel C, Thorpe P, Da Rocha M, Bajew S, Neilson R, Sokolova E, Da Silva C, Guy J. The transcriptomes of Xiphinema index and Longidorus elongatus suggest independent acquisition of some plant parasitism genes by horizontal gene transfer in early-branching nematodes. Genes. 2017;8(10):287.

40. van Megen $H$, van den Elsen $S$, Holterman M, Karssen G, Mooyman P, Bongers T, Holovachov O, Bakker J, Helder J. A phylogenetic tree of nematodes based on about 1200 full-length small subunit ribosomal DNA sequences. Nematology. 2009;1 1(6):927-50.

41. Holterman M, Karegar A, Mooijman P, van Megen $\mathrm{H}$, van den Elsen S, Vervoort MT, Quist CW, Karssen G, Decraemer W, Opperman CH. Disparate gain and loss of parasitic abilities among nematode lineages. PLoS One. 2017;12(9):e0185445

42. Hotopp JCD. Horizontal gene transfer between bacteria and animals. Trends Genet. 2011;27(4):157-63.

43. Bass C, Field LM. Gene amplification and insecticide resistance. Pest Manas Sci. 2011;67(8):886-90.

44. Kondrashov FA. Gene duplication as a mechanism of genomic adaptation to a changing environment. In: Proc R Soc B: 2012: The Royal Society; 2012. p. 5048-57.

45. Daron J, Glover N, Pingault L, Theil S, Jamilloux V, Paux E, Barbe V, Mangenot S, Alberti A, Wincker P. Organization and evolution of transposable elements along the bread wheat chromosome 3B. Genome Biol. 2014;15(12):546.

46. Vanholme B, Kast P, Haegeman A, Jacob J, Grunewald W, Gheysen G. Structural and functional investigation of a secreted chorismate mutase from the plant-parasitic nematode Heterodera schachtii in the context of related enzymes from diverse origins. Mol Plant Pathol. 2009;10(2):189-200.

47. De Boer J, Yan Y, Smant G, Davis E, Baum T. In-situ hybridization to messenger RNA in Heterodera glycines. J Nematol. 1998;30(3):309.

48. Wu TD, Reeder J, Lawrence M, Becker G, Brauer MJ. GMAP and GSNAP for genomic sequence alignment: enhancements to speed, accuracy, and functionality. In: Statistical Genomics: Methods and Protocols; 2016. p. 283-334
49. Huang X, Madan A. CAP3: a DNA sequence assembly program. Genome Res. 1999;9(9):868-77.

50. Wick RR, Schultz MB, Zobel J, Holt KE. Bandage: interactive visualization of de novo genome assemblies. Bioinformatics. 2015;31(20):3350-2.

51. Seo J-S, Rhie A, Kim J, Lee S, Sohn M-H, Kim C-U, Hastie A, Cao H, Yun $J$-Y, Kim J. De novo assembly and phasing of a Korean human genome. Nature. 2016.

52. Makoff AJ, Flomen RH. Detailed analysis of 15q11-q14 sequence corrects errors and gaps in the public access sequence to fully reveal large segmental duplications at breakpoints for Prader-Willi, Angelman, and inv dup (15) syndromes. Genome Biol. 2007;8(6):R114.

53. Li H, Durbin R. Fast and accurate short read alignment with burrowswheeler transform. Bioinformatics. 2009:25(14):1754-60.

54. Chaisson MJ, Tesler G. Mapping single molecule sequencing reads using basic local alignment with successive refinement (BLASR): application and theory. BMC Bioinf. 2012;13(1):238.

55. Kumar S, Jones M, Koutsovoulos G, Clarke M, Blaxter M. Blobology: exploring raw genome data for contaminants, symbionts and parasites using taxonannotated GC-coverage plots. Front Genet. 2013;4:237.

56. Hoff KJ, Lange S, Lomsadze A, Borodovsky M, Stanke M. BRAKER1: unsupervised RNA-Seq-based genome annotation with GeneMark-ET and AUGUSTUS. Bioinformatics. 2015;32(5):767-9.

57. Kim D, Langmead B, Salzberg SL. HISAT: a fast spliced aligner with low memory requirements. Nat Methods. 2015;12(4):357-60.

58. Wu TD, Nacu S. Fast and SNP-tolerant detection of complex variants and splicing in short reads. Bioinformatics. 2010;26(7):873-81.

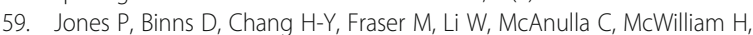
Maslen J, Mitchell A, Nuka G. InterProScan 5: genome-scale protein function classification. Bioinformatics. 2014;30(9):1236-40.

60. Hawkins JS, Kim H, Nason JD, Wing RA, Wendel JF. Differential lineagespecific amplification of transposable elements is responsible for genome size variation in Gossypium. Genome Res. 2006;16(10):1252-61.

61. Conesa A, Götz S, García-Gómez JM, Terol J, Talón M, Robles M. Blast2GO: a universal tool for annotation, visualization and analysis in functional genomics research. Bioinformatics. 2005;21(18):3674-6.

62. Altschul SF, Gish W, Miller W, Myers EW, Lipman DJ. Basic local alignment search tool. J Mol Biol. 1990;215(3):403-10.

63. Consortium U. UniProt: the universal protein knowledgebase. Nucleic Acids Res. 2017;45(D1):D158-69.

64. Suzek BE, Huang H, McGarvey P, Mazumder R, Wu CH. UniRef: comprehensive and non-redundant UniProt reference clusters. Bioinformatics. 2007;23(10):1282-8.

65. Smit AFA, Hubley R. RepeatModeler Open-1.0 (2008-2015). http://www. repeatmasker.org

66. Smit A, Hubley R, Green P: RepeatMasker Open-4.0. 2013-2015. Institute for Systems Biology http://repeatmasker org 2015.

67. Xiong W, He L, Lai J, Dooner HK, Du C. HelitronScanner uncovers a large overlooked cache of Helitron transposons in many plant genomes. Proc Natl Acad Sci. 2014;111(28):10263-8.

68. Heinz S, Benner C, Spann N, Bertolino E, Lin YC, Laslo P, Cheng JX, Murre C, Singh $\mathrm{H}$, Glass CK. Simple combinations of lineage-determining transcription factors prime cis-regulatory elements required for macrophage and B cell identities. Mol Cell. 2010:38(4):576-89.

69. Grant CE, Bailey TL, Noble WS. FIMO: scanning for occurrences of a given motif. Bioinformatics. 2011;27(7):1017-8.

70. Gao B, Allen R, Maier T, Davis EL, Baum TJ, Hussey RS. Identification of putative parasitism genes expressed in the esophageal gland cells of the soybean cyst nematode Heterodera glycines. Mol Plant-Microbe Interact. 2001;14(10):1247-54

71. Wang X, Allen R, Ding X, Goellner M, Maier T, de Boer JM, Baum TJ, Hussey RS, Davis EL. Signal peptide-selection of CDNA cloned directly from the esophageal gland cells of the soybean cyst nematode Heterodera glycines. Mol Plant-Microbe Interact. 2001;14(4):536-44.

72. Bailey TL, Johnson J, Grant CE, Noble WS. The MEME suite. Nucleic Acids Res. 2015;43(W1):W39-49.

73. Cotton JA, Lilley CJ, Jones LM, Kikuchi T, Reid AJ, Thorpe P, Tsai IJ, Beasley H, Blok V, Cock PJ. The genome and life-stage specific transcriptomes of Globodera pallida elucidate key aspects of plant parasitism by a cyst nematode. Genome Biol. 2014;15(3):R43.

74. Opperman CH, Bird DM, Williamson VM, Rokhsar DS, Burke M, Cohn J, Cromer J, Diener S, Gajan J, Graham S, et al. Sequence and genetic map of 
Meloidogyne hapla: a compact nematode genome for plant parasitism. Proc Natl Acad Sci. 2008;105(39):14802-7.

75. Howe KL, Bolt BJ, Cain S, Chan J, Chen WJ, Davis P, Done J, Down T, Gao S, Grove C. WormBase 2016: expanding to enable helminth genomic research. Nucleic Acids Res. 2015; gkv1217.

76. Phillips WS, Howe DK, Brown AM, Eves-Van Den Akker S, Dettwyler L, Peetz $A B$, Denver DR, Zasada IA. The Draft Genome of Globodera ellingtonae. J Nematol. 2017:49(2):127.

77. Drillon G, Carbone A, Fischer G. SynChro: a fast and easy tool to reconstruct and visualize synteny blocks along eukaryotic chromosomes. PLoS One. 2014;9(3):e92621.

78. Sulston J, Du Z, Thomas K, Wilson R, Hillier L, Staden R, Halloran N, Green P, Thierry-Mieg J, Qiu L. The C. elegans genome sequencing project: a beginning. Nature. 1992;356(6364):37.

79. Opperman CH, Bird DM, Williamson VM, Rokhsar DS, Burke M, Cohn J, Cromer J, Diener S, Gajan J, Graham S. Sequence and genetic map of Meloidogyne hapla: a compact nematode genome for plant parasitism. Proc Natl Acad Sci. 2008;105(39):14802-7.

80. Proost S, Fostier J, De Witte D, Dhoedt B, Demeester P, Van de Peer $Y$, Vandepoele K. I-ADHoRe 3.0-fast and sensitive detection of genomic homology in extremely large data sets. Nucleic Acids Res. 2011;40(2):e11.

81. Krzywinski M, Schein J, Birol I, Connors J, Gascoyne R, Horsman D, Jones SJ, Marra MA. Circos: an information aesthetic for comparative genomics. Genome Res. 2009;19(9):1639-45.

82. Löytynoja A. Phylogeny-aware alignment with PRANK. In: Multiple sequence alignment methods; 2014. p. 155-70.

83. Lee C, Yu D, Choi H-K, Kim RW. Reconstruction of a composite comparative map composed of ten legume genomes. Genes Genomics. 2017;39(1):111-9.

84. Stamatakis A. RAxML version 8: a tool for phylogenetic analysis and postanalysis of large phylogenies. Bioinformatics. 2014;30(9):1312-3.

85. Mirarab S, Reaz R, Bayzid MS, Zimmermann T, Swenson MS, Warnow T. ASTRAL genomescale coalescent-based species tree estimation. Bioinformatics. 2014;30(17):541-8.

86. Audemard E, Schiex T, Faraut T. Detecting long tandem duplications in genomic sequences. BMC Bioinf. 2012;13(1):83.

87. Coordinators NR. Database resources of the national center for biotechnology information. Nucleic Acids Res. 2016;44(Database issue):D7.

88. Andrews S. FastQC: a quality control tool for high throughput sequence data; 2010.

89. Quinlan AR. BEDTools: the Swiss-army tool for genome feature analysis. In: Current protocols in bioinformatics; 2014. 11.12. 11-11.12. 34.

90. Browning BL, Browning SR. Genotype imputation with millions of reference samples. Am J Hum Genet. 2016;98(1):116-26.

91. Browning SR, Browning BL. Rapid and accurate haplotype phasing and missing-data inference for whole-genome association studies by use of localized haplotype clustering. Am J Hum Genet. 2007;81(5):1084-97.

92. Zheng X, Levine D, Shen J, Gogarten SM, Laurie C, Weir BS. A highperformance computing toolset for relatedness and principal component analysis of SNP data. Bioinformatics. 2012;28(24):3326-8.

93. Liao Y, Smyth GK. Shi W: featureCounts: an efficient general purpose program for assigning sequence reads to genomic features. Bioinformatics. 2013;30(7):923-30.

94. Love Ml, Huber W, Anders S. Moderated estimation of fold change and dispersion for RNA-seq data with DESeq2. Genome Biol. 2014;15(12):550.

95. Merino GA, Conesa A, Fernández EA. A benchmarking of workflows for detecting differential splicing and differential expression at isoform level in human RNA-seq studies. Brief Bioinform. 2017.

96. Bolger AM, Lohse M, Usadel B. Trimmomatic: a flexible trimmer for Illumina sequence data. Bioinformatics. 2014;30(15):2114-20.

97. Kim D, Pertea G, Trapnell C, Pimentel H, Kelley R, Salzberg SL. TopHat2: accurate alignment of transcriptomes in the presence of insertions, deletions and gene fusions. Genome Biol. 2013;14(4):R36.

98. Trapnell C, Roberts A, Goff L, Pertea G, Kim D, Kelley DR, Pimentel H, Salzberg SL, Rinn JL, Pachter L. Differential gene and transcript expression analysis of RNA-seq experiments with TopHat and cufflinks. Nat Protoc. 2012;7(3):562.

99. Pachter L: Models for transcript quantification from RNA-Seq. arXiv preprint arXiv:11043889 2011.

100. Vitting-Seerup K, Sandelin A. The landscape of isoform switches in human cancers. Mol Cancer Res. 2017;15(9):1206-20.

101. Finn RD, Attwood TK, Babbitt PC, Bateman A, Bork P, Bridge AJ, Chang H-Y, Dosztányi Z, El-Gebali S, Fraser M. InterPro in 2017-beyond protein family and domain annotations. Nucleic Acids Res. 2016:45(D1):D190-9.
102. Towns J, Cockerill T, Dahan M, Foster I, Gaither K, Grimshaw A, Hazlewood V, Lathrop S, Lifka D, Peterson GD. XSEDE: accelerating scientific discovery. Comput Sci Eng. 2014;16(5):62-74.

103. Nystrom NA, Levine MJ, Roskies RZ, Scott J. Bridges: a uniquely flexible HPC resource for new communities and data analytics. In: Proceedings of the 2015 XSEDE Conference: Scientific Advancements Enabled by Enhanced Cyberinfrastructure: 2015: ACM; 2015. p. 30.

\section{Ready to submit your research? Choose BMC and benefit from:}

- fast, convenient online submission

- thorough peer review by experienced researchers in your field

- rapid publication on acceptance

- support for research data, including large and complex data types

- gold Open Access which fosters wider collaboration and increased citations

- maximum visibility for your research: over $100 \mathrm{M}$ website views per year

At BMC, research is always in progress.

Learn more biomedcentral.com/submissions 